\title{
Comparative effects of telmisartan and valsartan as add-on agents for hypertensive patients with morning blood pressure insufficiently controlled by amlodipine monotherapy
}

\author{
Hideaki Yoshida $^{1}$, Hiroshi Akasaka ${ }^{1}$, Shigeyuki Saitoh ${ }^{1}$, Kazuaki Shimamoto ${ }^{1}$ and Tetsuji Miura ${ }^{1}$ on behalf of \\ the SPEED investigators ${ }^{2}$
}

The aim of this study was to determine the efficacies of valsartan and telmisartan as add-on agents for the control of morning blood pressure (BP) in patients already on amlodipine monotherapy. A total of 414 hypertensive patients were prospectively enrolled in a 4-week run-in period when they were treated with amlodipine $(5 \mathrm{mg} / \mathrm{day})$, and home BP was measured in the morning and evening. Patients with home systolic BP (SBP) being $135-159 \mathrm{~mm} \mathrm{Hg}$ in the morning at the end of the run-in period were randomized to additional treatment with valsartan $(80 \mathrm{mg} /$ day $)$ or with telmisartan $(40 \mathrm{mg} /$ day $)$ for 8 weeks. The primary endpoint was the change in morning home BP, and secondary endpoints included variability of morning home BP. Of the 282 patients randomized, 262 patients $(n=131$, in each treatment) completed the protocols. Demographic parameters and baseline morning SBP/diastolic BP (DBP) $(146.3 \pm 7.1 / 84.8 \pm 9.3$ vs. $146.0 \pm 7.1 / 84.2 \pm 9.1 \mathrm{~mm} \mathrm{Hg})$ were comparable in the valsartan group and telmisartan group, and changes in SBP/DBP after 8-week treatment were not significantly different between the two groups $(-7.4 \pm 10.6 /-3.9 \pm 6.1$ vs. $-8.3 \pm 9.9 /-5.0 \pm 5.9 \mathrm{~mm} \mathrm{Hg})$. Valsartan significantly increased individual standard deviation and variation coefficient of morning SBP, but telmisartan did not change either of these indices of SBP variation. In subgroups with baseline SBP being above the median $(145.2 \mathrm{~mm} \mathrm{Hg})$, change in DBP was significantly larger by telmisartan than by valsartan $(-6.3 \pm 5.6$ vs. $-3.9 \pm 6.7 \mathrm{~mm} \mathrm{Hg}, P<0.05)$. These results suggest that telmisartan is more useful than valsartan as an add-on agent for reducing the level and variability of morning BP in patients on amlodipine monotherapy.

Hypertension Research (2014) 37, 225-231; doi:10.1038/hr.2013.141; published online 10 October 2013

Keywords: blood pressure variation; telmisartan; valsaratan

\section{INTRODUCTION}

Accumulating evidence indicates that levels of blood pressure (BP) determined by ambulatory BP monitoring and home BP monitors are better predictors than office BP of cardiovascular events. ${ }^{1-3}$ In addition to the BP level, variability of $\mathrm{BP}$ has been shown to significantly influence the clinical outcomes of hypertensive patients. Not only circadian variation of BP but also visit-to-visit variation in $\mathrm{BP}$ and day-by-day variation in home BP were shown to be associated with an increased risk of cardiovascular mortality in studies using ambulatory BP monitoring and home BP monitoring, ${ }^{4-9}$ although the impact of 'morning surge' of BP on prognosis remains controversial. $^{1-12}$ Currently, long-acting antihypertensive agents are widely used for 24-h control of BP. However, recent studies have disclosed that BP during the early-morning hours remains high in a large number of hypertensive patients on treatment. ${ }^{13-15}$ Furthermore, inter-individual variation in BP response differs between classes of anti-hypertensive agents, ${ }^{16}$ and it is likely that such inter-individual variation is also present within a class of agents owing to different pharmacokinetic features of each specific agent. Thus, it has not been established which combination of antihypertensive drugs is suitable to sufficiently control both average and variation of BP during a day.

In the present study, we compared the effects of valsartan and telmisartan as add-on agents on morning BP and variation in BP for control of $\mathrm{BP}$ in hypertensive patients already being treated with a calcium channel blocker (CCB). The rationale for this study is threefold. First, a long-acting CCB is an anti-hypertensive agent for which inter-individual variation in SBP is smaller than that of other

\footnotetext{
${ }^{1}$ Department of Cardiovascular, Renal and Metabolic Medicine, Sapporo Medical University, School of Medicine, Sapporo, Japan 2See Appendix.

Correspondence: Dr T Miura, Department of Cardiovascular, Renal and Metabolic Medicine, Sapporo Medical University School of Medicine, South-1 West-16, Chuo-ku, Sapporo, 060-8556, Japan.

E-mail: miura@sapmed.ac.jp

Received 20 May 2013; revised 2 August 2013; accepted 22 August 2013; published online 10 October 2013
} 
agents and is thus a good choice as a baseline treatment. ${ }^{16}$ Second, an angiotensin receptor antagonist (ARB) is preferable to diuretics as an agent to combine with CCB from a metabolic point of view. Third, plasma half-lives of valsartan and telmisartan are different $(6-10 \mathrm{~h}$ vs. $21-38 \mathrm{~h}),{ }^{17}$ which possibly results in difference in levels of morning BP control. Although head-to-head comparison of telmisartan and valsartan as monotherapy has been reported previously, ${ }^{18-20}$ comparative effects of these ARBs as agents in combination with a CCB have not been examined. We addressed this issue by a prospective randomized open-label blinded-endpoint (PROBE) study design with morning home BP as a primary endpoint.

\section{METHODS}

The present study was conducted at 36 clinical sites that participated in the SPEED (Sapporo study to prove efficacies of telmisartan and valsartan for early-morning blood pressure as add-on therapy) study. The protocol of this study was approved by the Clinical Investigation Ethics Committee of Sapporo Medical University Hospital and was registered in the UMIN Clinical Trials Registry (UMIN 000003922). We conducted this study in strict adherence with the principles of the Declaration of Helsinki. Written informed consent was obtained from all study participants.

\section{Study design and subjects}

This study was designed as a PROBE trial. As shown in Figure 1, hypertensive patients at the age of 40-79 years were enrolled in a 4week run-in period when amlodipine $(5 \mathrm{mg} /$ day) was administered, and home BP was determined in the early morning (within $1 \mathrm{~h}$ after waking up) and in the evening (within $1 \mathrm{~h}$ before sleeping) with a semi-automated sphygmomanometer (HEM-7080IC, OMRON HEALTHCARE, Kyoto, Japan). Patients whose home SBP in the morning was $135 \mathrm{~mm} \mathrm{Hg}$ or higher but lower than $160 \mathrm{~mm} \mathrm{Hg}$ were randomized to a valsartan $(80 \mathrm{mg} /$ day $)$ group or to a telmisartan ( $40 \mathrm{mg} /$ day) group for an 8-week add-on therapy. Randomization of all subjects was performed by a central registration office. Exclusion criteria were allergy to telmisartan or valsartan, pregnancy, severe liver dysfunction, serum creatinine $\geqslant 3.0 \mathrm{mg} \mathrm{dl}^{-1}$, malignant hypertension and secondary hypertension. Patients with chronic heart failure, atrial fibrillation, history of myocardial infarction, proteinuria, diabetes mellitus or metabolic syndrome were also excluded from the

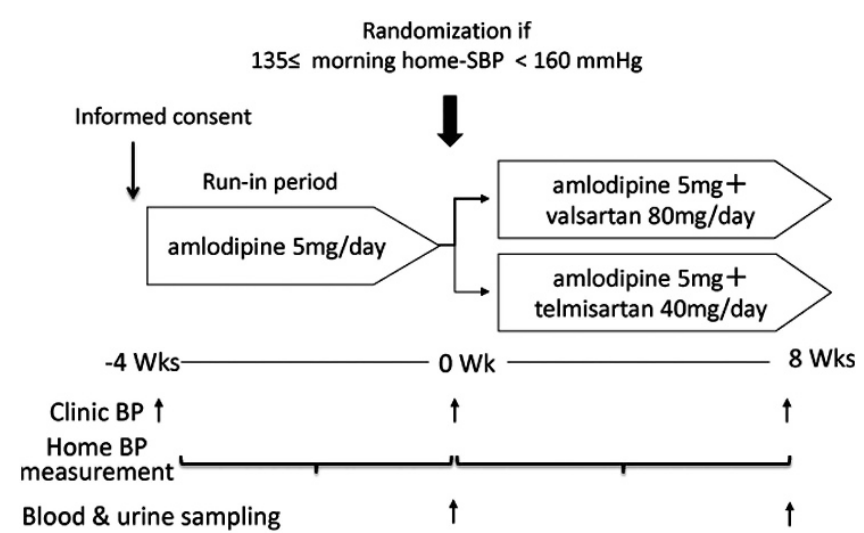

Figure 1 Study design. BP data were retrieved from semiautomated sphygmomanometers at the time of randomization ( $0 \mathrm{Wk}$ ) and at 8 weeks after ARB treatments ( 8 Wks). BP values within 5 days before randomization and those within 5 days before the end of the ARB treatment period were used for calculation of average BP and BP variability indices. enrollment in the run-in period, as CCB is not the first choice for these patients in Treatment Guidelines of the Japanese Society of Hypertension 2009 (The Japanese Society of Hypertension:guidelines for the management of hypertension, http://www.jpnsh.org/guideline.html). The primary endpoint was change in home SBP in the early morning, and secondary endpoints were variability of home SBP in the early morning and home BP before sleep.

On the basis of the results of a previous study showing that morning home BP differed by $2.3 \mathrm{~mm} \mathrm{Hg}$ in patients treated with 40 $80 \mathrm{mg} /$ day of telmisartan and those treated with $80-160 \mathrm{mg} /$ day of valsartan ${ }^{21}$ and the assumption that standard deviation of home BP is approximately $6 \mathrm{~mm} \mathrm{Hg}$, we estimated that a sample size of 125 patients per treatment arm would be necessary to detect a $2.3 \mathrm{~mm} \mathrm{Hg}$ difference between the two treatment groups with $80 \%$ power.

\section{Determination of BP and its variability}

After enrollment, study subjects were requested to measure BP by the HEM-7080IC within $1 \mathrm{~h}$ after waking up and before sleeping every day, and BP data were automatically stored in the HEM-7080IC with a $14.6 \times 44.6-\mathrm{cm}$ arm cuff. The method of BP measurement (that is, BP measurement by use of the HEM-7080IC placed at the level of the heart in a sitting position with feet on the floor after a 2-min rest following urination) was explained to all study participants using written instructions with illustrations. BP data were retrieved at the time of randomization and at 8 weeks after ARB treatments, and the first BP data and the last BP data within a day were used as morning home BP and home BP before sleep, respectively, for that day. BP values within 5 days before randomization and those within 5 days before the end of the ARB treatment period were used for calculation of BP and its variability. Standard deviation (s.d.) of BP during a 5 -day period and coefficient of variation $(\mathrm{CV}$, s.d. divided by mean $\mathrm{BP}$ ) were used as indices of $\mathrm{BP}$ variability. Office $\mathrm{BP}$ data at baseline and 8 weeks after ARB treatments were collected from each clinic together with the data for demographic parameters.

\section{Biochemical evaluation}

Blood and urine samples were obtained at the time of randomization for biochemical analyses in a central laboratory (Daiichi-Kishimoto Clinical Laboratory, Sapporo, Japan). The estimated glomerular filtration rate was calculated from the data for serum creatinine, age and sex by the use of equations for Japanese. ${ }^{22}$

\section{Statistical analysis}

Data were analyzed by intention-to-treat analysis. Group mean data are expressed as means \pm s.d. Differences between means of parameters in treatment groups and differences between means within a group were tested by Student's $t$-test and by Student's paired $t$-test, respectively. Inter-group differences in percentages of demographic parameters were examined by the $\chi^{2}$-test. Correlations of two parameters were assessed by the use of Pearson's correlation coefficient. A difference was considered to be statistically significant if the $P$-value was $<0.05$. All statistical analyses were performed using SPSS statistics 20 (IBM, NY, USA).

\section{RESULTS}

Enrollment and disposition of patients

During a 4-week run-in period, 414 hypertensive patients were enrolled and 282 of those patients were eligible for randomization (that is, $135 \leqslant$ home SBP in the morning $<160 \mathrm{~mm} \mathrm{Hg}$ ) (Figure 2). After randomization, 20 patients dropped out from the protocol because of adverse events (general malaise or tinnitus: 3 patients in 


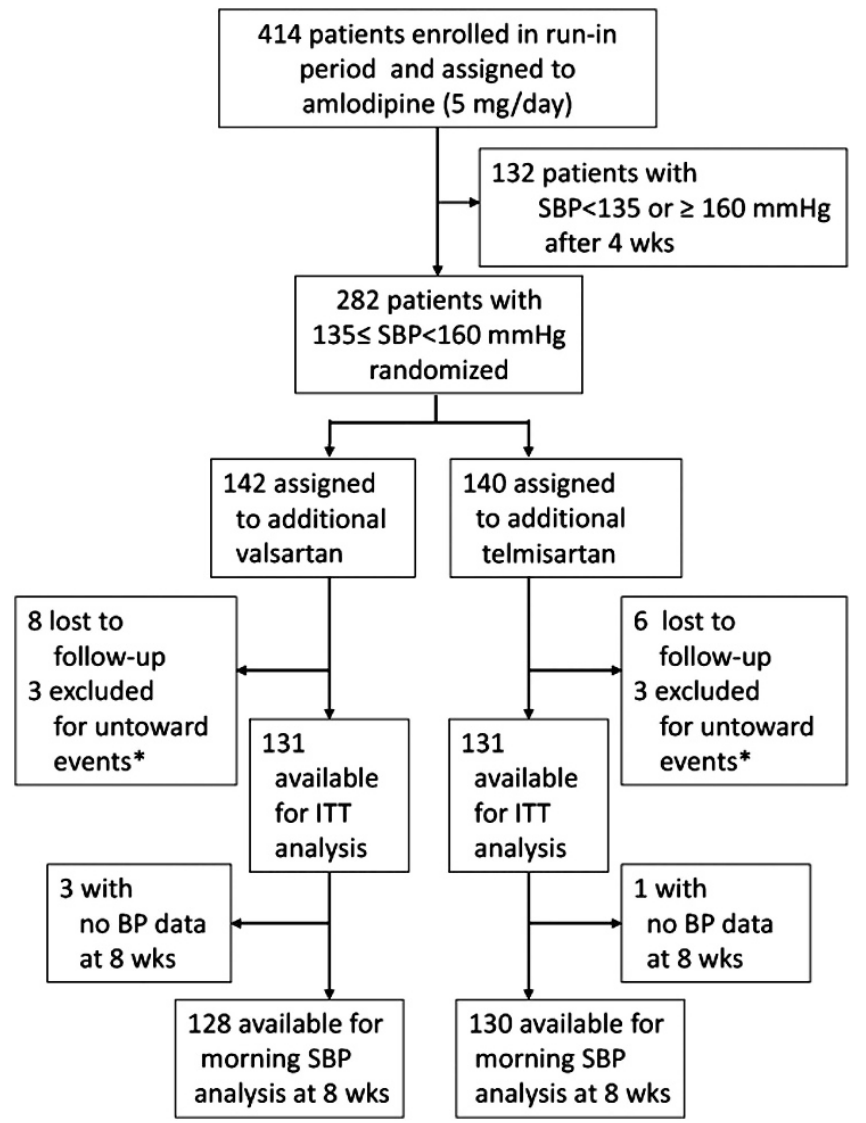

Figure 2 Patient disposition. *Untoward events, general malaise or tinnitus; wks, weeks; ITT, intention-to-treat.

each treatment arm) or for no show in the clinic during the follow-up period ( 8 patients in the valsartan group and 6 patients in the telmisartan group). Thus, 262 patients (131 patients in each treatment arm) completed the 8-week protocol of ARB treatment and contributed to the intention-to-treat analysis. Of the 262 randomized patients, 4 subjects ( 3 in the valsartan group and 1 in the telmisartan group) were found to lack recordings of home BP at the end of the 8week ARB treatment period. Accordingly, 258 patients contributed to analysis of the primary endpoint.

Clinical characteristics of the study subjects

Demographic parameters in subjects are shown in Table 1. Age, male percentage, body mass index (BMI) and abdominal circumference were comparable between the valsartan-treated and telmisartantreated groups, and the duration of hypertension and incidences of comorbidities were also similar in the two groups. Baseline serum and urine chemistry data are shown in Table 2. There was no significant difference between the valsartan and telmisartan groups in serum lipids, glucose and glycoalbumin or renal function indices, including estimated glomerular filtration rate. Clinical characteristics were comparable between 128 valsartan-treated and 130 telmisartantreated patients in whom morning BP data after 8 weeks of ARB treatment were available (data not shown).

\section{Home BP data}

As shown in Table 3, baseline SBP and DBP early in the morning were comparable between the valsartan and telmisartan groups (SBP/
Table 1 Demographic characteristics

\begin{tabular}{|c|c|c|c|}
\hline & $\begin{array}{l}\text { Valsartan } \\
(\mathrm{n}=131)\end{array}$ & $\begin{array}{c}\text { Telmisartan } \\
(\mathrm{n}=131)\end{array}$ & P-value \\
\hline Age (years) & $61.2 \pm 10.6$ & $62.2 \pm 10.0$ & 0.444 \\
\hline Male & 66 (50.4\%) & 64 (48.9\%) & 0.805 \\
\hline Height (cm) & $159.6 \pm 9.3$ & $159.6 \pm 10.1$ & 0.984 \\
\hline Weight (kg) & $62.6 \pm 10.9$ & $62.3 \pm 11.5$ & 0.851 \\
\hline BMI $\left(\mathrm{kg} \mathrm{m}^{-2}\right)$ & $24.5 \pm 3.6$ & $24.4 \pm 3.2$ & 0.827 \\
\hline Abdominal circumference $(\mathrm{cm})$ & $84.5 \pm 10.3$ & $84.8 \pm 9.3$ & 0.808 \\
\hline Current smoker & $32(24.4 \%)$ & $29(22.1 \%)$ & 0.686 \\
\hline Current drinker & $59(45.0 \%)$ & $63(48.1 \%)$ & 0.748 \\
\hline \multicolumn{4}{|l|}{ Duration of hypertension (years) } \\
\hline$<1$ & $41(31.3 \%)$ & $43(32.8 \%)$ & 0.715 \\
\hline$\geqslant 1-<5$ & $38(29.0 \%)$ & $33(25.2 \%)$ & \\
\hline$\geqslant 5-<10$ & $20(15.3 \%)$ & $20(15.3 \%)$ & \\
\hline$\geqslant 10$ & $15(11.5 \%)$ & $21(16.0 \%)$ & \\
\hline \multicolumn{4}{|l|}{ Comorbidities } \\
\hline Dyslipidemia & $32(24.4 \%)$ & $35(26.7 \%)$ & 0.671 \\
\hline Diabetes & $7(5.3 \%)$ & $5(3.8 \%)$ & 0.554 \\
\hline Hyperuricemia & $5(3.8 \%)$ & $3(2.3 \%)$ & 0.473 \\
\hline Cerebral hemorrhage & $1(0.8 \%)$ & $1(0.8 \%)$ & 1.000 \\
\hline Cerebral infarction & $2(1.5 \%)$ & $1(0.8 \%)$ & 0.561 \\
\hline Myocardial infarction & $0(0 \%)$ & $3(2.3 \%)$ & 0.081 \\
\hline Angina pectoris & $3(2.3 \%)$ & $4(3.1 \%)$ & 0.702 \\
\hline Heart failure & $0(0 \%)$ & $1(0.8 \%)$ & 0.316 \\
\hline Atrial fibrillation & $0(0 \%)$ & $1(0.8 \%)$ & 0.316 \\
\hline Chronic kidney disease & $2(1.5 \%)$ & $0(0 \%)$ & 0.156 \\
\hline Peripheral artery disease & $2(1.5 \%)$ & $0(0 \%)$ & 0.156 \\
\hline
\end{tabular}

Table 2 Baseline laboratory data

\begin{tabular}{|c|c|c|c|}
\hline & $\begin{array}{l}\text { Valsartan } \\
(n=130)\end{array}$ & $\begin{array}{l}\text { Telmisartan } \\
(\mathrm{n}=131)\end{array}$ & P-value \\
\hline Total cholesterol (mg dl-1) & $209.2 \pm 34.8$ & $210.9 \pm 32.5$ & 0.685 \\
\hline Triglyceride $\left(\mathrm{mg} \mathrm{dl}^{-1}\right)$ & $154.6 \pm 167.1$ & $145.8 \pm 97.5$ & 0.605 \\
\hline HDL cholesterol (mg dl ${ }^{-1}$ ) & $61.3 \pm 17.7$ & $60.8 \pm 15.7$ & 0.804 \\
\hline Blood glucose (mg dl $\left.{ }^{-1}\right)$ & $102.5 \pm 26.0$ & $100.5 \pm 19.2$ & 0.480 \\
\hline Glycoalbumin (\%) & $14.3 \pm 1.7$ & $14.5 \pm 1.7$ & 0.366 \\
\hline Insulin $\left(\mu \mathrm{U} \mathrm{ml}^{-1}\right)$ & $14.3 \pm 20.7$ & $12.3 \pm 12.8$ & 0.345 \\
\hline Serum creatinine $\left(\mathrm{mg} \mathrm{dl}^{-1}\right)$ & $0.64 \pm 0.14$ & $0.66 \pm 0.13$ & 0.296 \\
\hline eGFR $(\mathrm{ml} \mathrm{min}-1)$ & $87.5 \pm 16.9$ & $84.1 \pm 16.3$ & 0.099 \\
\hline$\gamma$-GTP $\left(I \cup \mathrm{ml}^{-1}\right)$ & $64.9 \pm 113.8$ & $59.2 \pm 82.0$ & 0.645 \\
\hline UACR (mg g ${ }^{-1}$ Cre) & $30.5 \pm 66.1$ & $32.4 \pm 52.9$ & 0.798 \\
\hline $\mathrm{HsCRP}\left(\mathrm{mg} \mathrm{dl}{ }^{-1}\right)$ & $0.15 \pm 0.53$ & $0.12 \pm 0.24$ & 0.583 \\
\hline
\end{tabular}

Abbreviations: HsCRP, high sense C-reactive peptide; UACR, urinary albumin/creatinine ratio. Mean \pm s.d.

$\mathrm{DBP}=146.3 \pm 7.1 / 84.8 \pm 9.3$ vs. $146.0 \pm 7.1 / 84.2 \pm 9.1 \mathrm{~mm} \mathrm{Hg})$. At 8 weeks after the start of treatment, both valsartan and telmisartan significantly reduced morning SBP in patients on amlodipine, and there was no significant difference in SBP $(138.9 \pm 12.0 \mathrm{vs}$. $137.7 \pm 10.4 \mathrm{~mm} \mathrm{Hg}) \quad$ or $\mathrm{DBP} \quad(80.9 \pm 9.7$ vs. $79.2 \pm 9.2 \mathrm{~mm} \mathrm{Hg})$ between the two groups. Changes in morning SBP and DBP tended to be larger in the telmisartan group than in the valsartan group $(\Delta \mathrm{SBP}=-8.3 \pm 9.9$ vs. $-7.4 \pm 10.6 \mathrm{~mm} \mathrm{Hg}, \Delta \mathrm{DBP}=-5.0 \pm 5.9$ vs. $-3.9 \pm 6.1 \mathrm{~mm} \mathrm{Hg}$ ), but the differences were not statistically significant. 
Table 3 Home BP and heart rate measurements early in the morning

\begin{tabular}{|c|c|c|c|c|c|c|c|c|c|}
\hline & \multicolumn{3}{|c|}{ All subjects } & \multicolumn{3}{|c|}{$S B P<145.2 \mathrm{~mm} \mathrm{Hg}$ at baseline } & \multicolumn{3}{|c|}{$S B P \geqslant 145.2 \mathrm{~mm} \mathrm{Hg}$ at baseline } \\
\hline & $\begin{array}{l}\text { Valsartan } \\
(\mathrm{n}=128)\end{array}$ & $\begin{array}{c}\text { Telmisartan } \\
(\mathrm{n}=130)\end{array}$ & P-value & $\begin{array}{l}\text { Valsartan } \\
(\mathrm{n}=65)\end{array}$ & $\begin{array}{c}\text { Telmisartan } \\
\quad(\mathrm{n}=65)\end{array}$ & P-value & $\begin{array}{l}\text { Valsartan } \\
(\mathrm{n}=63)\end{array}$ & $\begin{array}{c}\text { Telmisartan } \\
\quad(\mathrm{n}=65)\end{array}$ & P-Value \\
\hline \multicolumn{10}{|l|}{$S B P(m m ~ H g)$} \\
\hline Baseline & $146.3 \pm 7.1$ & $146.0 \pm 7.1$ & 0.716 & $140.4 \pm 3.4$ & $139.8 \pm 2.9$ & 0.244 & $152.3 \pm 4.2$ & $152.1 \pm 4.0$ & 0.814 \\
\hline 8-weeks & $138.9 \pm 12.0$ & $137.7 \pm 10.4$ & 0.385 & $134.4 \pm 10.7$ & $134.0 \pm 9.2$ & 0.823 & $143.6 \pm 11.5$ & $141.4 \pm 10.3$ & 0.260 \\
\hline Change from baseline & $-7.4 \pm 10.6$ & $-8.3 \pm 9.9$ & 0.484 & $-6.1 \pm 11.0$ & $-5.8 \pm 9.5$ & 0.884 & $-8.7 \pm 10.1$ & $-10.7 \pm 9.8$ & 0.254 \\
\hline \multicolumn{10}{|l|}{$D B P(m m ~ H g)$} \\
\hline Baseline & $84.8 \pm 9.3$ & $84.2 \pm 9.1$ & 0.659 & $82.1 \pm 8.5$ & $81.9 \pm 8.6$ & 0.861 & $87.4 \pm 9.3$ & $86.6 \pm 9.1$ & 0.610 \\
\hline 8-weeks & $80.9 \pm 9.7$ & $79.2 \pm 9.2$ & 0.167 & $78.3 \pm 8.3$ & $78.2 \pm 9.2$ & 0.962 & $83.6 \pm 10.4$ & $80.3 \pm 9.2$ & 0.060 \\
\hline Change from baseline & $-3.9 \pm 6.1$ & $-5.0 \pm 5.9$ & 0.133 & $-3.9 \pm 5.6$ & $-3.7 \pm 6.0$ & 0.854 & $-3.9 \pm 6.7$ & $-6.3 \pm 5.6$ & 0.025 \\
\hline \multicolumn{10}{|l|}{$H R\left(\right.$ beat $\left.\mathrm{min}^{-1}\right)$} \\
\hline Baseline & $69.2 \pm 9.8$ & $68.0 \pm 10.1$ & 0.322 & $67.8 \pm 9.2$ & $68.2 \pm 11.0$ & 0.799 & $70.7 \pm 10.2$ & $67.7 \pm 9.2$ & 0.087 \\
\hline 8-weeks & $68.3 \pm 10.3$ & $67.3 \pm 9.3$ & 0.441 & $66.0 \pm 9.6$ & $67.8 \pm 10.7$ & 0.322 & $70.6 \pm 10.6$ & $66.8 \pm 7.8$ & 0.025 \\
\hline Change from baseline & $-0.9 \pm 5.4$ & $-0.7 \pm 4.6$ & 0.651 & $-1.7 \pm 4.7$ & $-0.4 \pm 4.4$ & 0.106 & $-0.1 \pm 5.9$ & $-0.9 \pm 4.8$ & 0.418 \\
\hline
\end{tabular}

Abbreviations: DBP, diastolic blood pressure; HR, heart rate; SBP, systolic blood pressure. Mean \pm s.d.
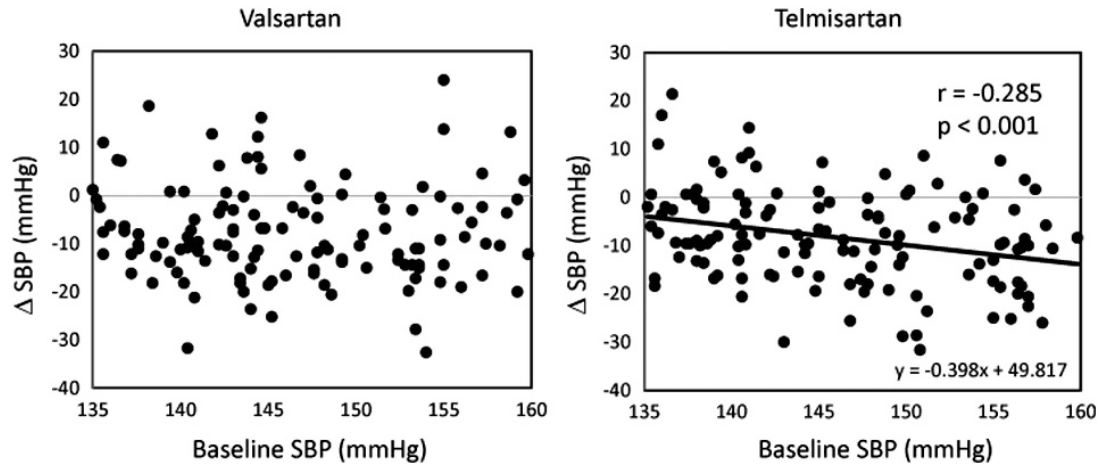

Figure 3 Relationship between baseline SBP and changes in SBP after the 8-week treatment. SBP $=$ home SBP early in the morning, $\triangle \mathrm{SBP}=\mathrm{change}$ in home SBP early in the morning after the 8-week treatment with valsartan or telmisartan.

Relationships between baseline SBP in subjects treated with amlodipine and changes in BP after addition of telmisartan or valsartan are shown in Figure 3. Change in SBP after addition of telmisartan was significantly correlated with baseline SBP $(r=-0.285, P<0.001)$, but such a correlation was not detected for valsartan treatment.

Figure 4 shows effects of valsartan and telmisartan on day-byday variation of home SBP early in the morning. Valsartan significantly increased individual s.d. and CV of SBP, whereas telmisartan did not significantly change either index of SBP variation.

Data on BP before sleep were missing in 55 subjects (26 in the valsartan group and 29 in the telmisartan group). Baseline SBP and DBP before sleep were comparable between the valsartan and telmisartan groups (SBP/ $\mathrm{DBP}=139.2 \pm 11.2 / 79.3 \pm 9.6$ vs. $137.6 \pm 11.1 / 77.7 \pm 9.0 \mathrm{~mm} \mathrm{Hg}$ ) (Table 4).
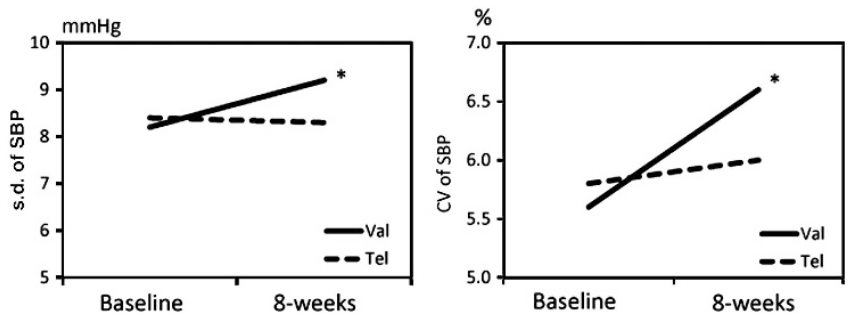

Figure 4 Effects of valsartan and telmisartan on day-by-day variation of SBP. Changes in s.d. of SBP early in the morning (left panel) and changes in CV of SBP early in the morning (right panel) are shown. Solid line and broken line indicate the valsartan group and telmisartan group, respectively. $\mathrm{SBP}=$ home SBP early in the morning, s.d., standard deviation, $\mathrm{CV}$, coefficient of variation, Val, valsartan group, Tel, telmisartan group. ${ }^{*} P<0.05$ vs. Baseline. 
Table 4 Home BP and heart rate before sleep

\begin{tabular}{lccc}
\hline & Valsartan $(\mathrm{n}=105)$ & Telmisartan $(\mathrm{n}=102)$ & P-value \\
\hline SBP $(\mathrm{mm} \mathrm{Hg})$ & & & \\
$\quad$ Baseline & $139.2 \pm 11.2$ & $137.6 \pm 11.1$ & 0.295 \\
8 weeks & $132.5 \pm 12.3$ & $129.7 \pm 11.9$ & 0.106 \\
Change from baseline & $-6.7 \pm 9.9$ & $-7.9 \pm 10.9$ & 0.440 \\
& & & \\
DBP (mm Hg) & & & 0.221 \\
Baseline & $79.3 \pm 9.6$ & $77.7 \pm 9.0$ & 0.060 \\
8 weeks & $75.3 \pm 10.4$ & $72.8 \pm 8.6$ & 0.319 \\
Change from baseline & $-4.0 \pm 6.6$ & $-4.9 \pm 6.5$ & \\
& & & 0.972 \\
HR (beat min $^{-1}$ ) & & & 0.900 \\
Baseline & $71.0 \pm 10.2$ & $71.0 \pm 9.5$ & 0.854 \\
8 weeks & $70.6 \pm 11.4$ & $-0.2 \pm 4.5$ & \\
Change from baseline & $-0.3 \pm 6.0$ & & \\
\hline
\end{tabular}

Abbreviations:DBP, diastolic blood pressure; HR, heart rate; SBP, systolic blood pressure. Mean \pm s.d.
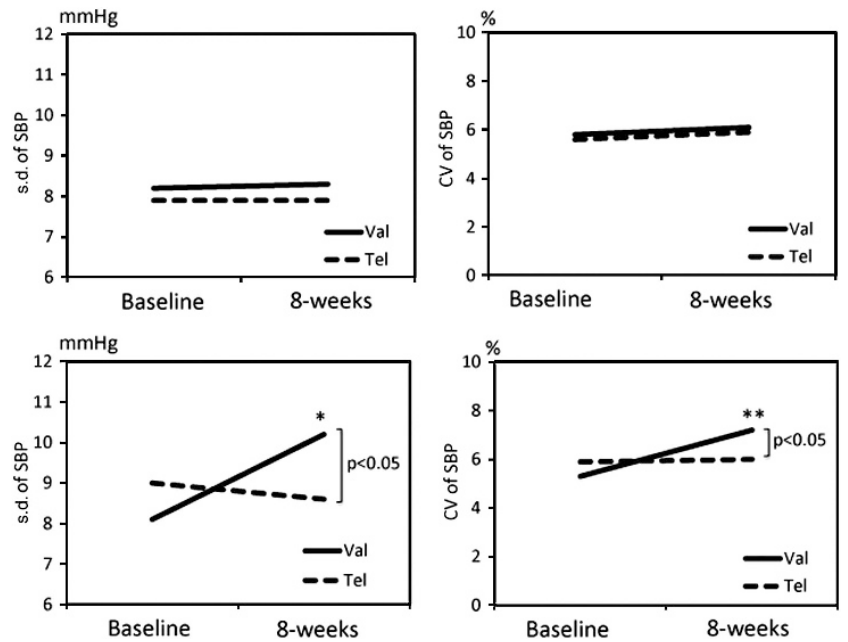

Figure 5 Effects of valsartan and telmisartan on home BP and variation of SBP: subgroup analyses. In subgroups with baseline $\mathrm{SBP}<145.2 \mathrm{~mm} \mathrm{Hg}$ (upper panels), there was no significant difference in changes in s.d. (left upper panel) or CV (right upper panel) of home BP early in the morning after the 8-week treatment between the valsartan and telmisartan subgroups. In subgroups with baseline SBP $\geqslant 145.2 \mathrm{~mm} \mathrm{Hg}$ (lower panels), s.d. (left lower panel) and CV (right lower panel) were significantly smaller in the telmisartan subgroup than in the valsartan subgroup. Val, valsartan subgroup, Tel, telmisartan subgroup. ${ }^{*} P<0.05$ vs. Baseline, ${ }^{*} P<0.01$ vs. Baseline.

BP levels before sleep at the end of the 8-week treatment period (SBP/ $\mathrm{DBP}=132.5 \pm 12.3 / 75.3 \pm 10.4$ vs. $129.7 \pm 11.9 / 72.8 \pm 8.6 \mathrm{~mm} \mathrm{Hg})$ or their changes after treatment $(\Delta \mathrm{SBP}=-6.7 \pm 9.9$ vs. $-7.9 \pm 10.9 \mathrm{~mm} \mathrm{Hg}$, $\Delta \mathrm{DBP}=-4.0 \pm 6.6 v s$. $-4.9 \pm 6.5 \mathrm{~mm} \mathrm{Hg})$ were not significantly different between the two groups.

\section{Subgroup analyses of home BP}

To examine possible differences in the effects of valsartan and telmisartan on SBP between subjects with low baseline SBP and those with high baseline SBP, we divided each treatment group into two subgroups by a median value of baseline SBP $(145.2 \mathrm{~mm} \mathrm{Hg})$. Demographic parameters were comparable between telmisartan- treated and valsartan-treated subjects within each SBP subgroup (data not shown).

There was no significant difference between the valsartan-treated and telmisartan-treated subgroups with baseline SBP being $<145.2$ $\mathrm{mm} \mathrm{Hg}$ in changes in SBP and DBP (Table 3), s.d. of SBP or CV of SBP (Figure 5, upper panels) after 8 weeks of treatment. However, in comparison with subgroups with baseline morning SBP $\geqslant 145.2 \mathrm{~mm}$ $\mathrm{Hg}$, reduction in DBP was significantly larger in telmisartan-treated patients than in valsartan-treated patients $(-6.3 \pm 5.6$ vs. $3.9 \pm 6.7 \mathrm{~mm} \mathrm{Hg}$ ), although changes in SBP were not significantly different (Table 3). Standard deviation and CV of SBP were increased by valsartan but not by telmisartan after 8 weeks of treatment in the subgroups with baseline SBP $\geqslant 145.2 \mathrm{~mm} \mathrm{Hg}$ (Figure 5, lower panels).

\section{Office BP data}

There was no significant difference between the valsartan group and the telmisartan group in office $\mathrm{BP}$ at baseline $(\mathrm{SBP} / \mathrm{DBP}=$ $143.8 \pm 11.9 / 83.1 \pm 8.4$ vs. $143.4 \pm 11.6 / 82.6 \pm 9.2 \mathrm{~mm} \mathrm{Hg})$ and 8 weeks after treatments $(134.6 \pm 11.4 / 78.2 \pm 9.2$ vs. $133.0 \pm 12.4 / 76.7 \pm$ $7.6 \mathrm{~mm} \mathrm{Hg})$. BP changes after treatments were also comparable in the two groups $(\triangle \mathrm{SBP}=-9.1 \pm 12.4$ vs. $-10.6 \pm 12.1 \mathrm{~mm} \mathrm{Hg}$, $\Delta \mathrm{DBP}=-4.9 \pm 7.5$ vs. $-6.0 \pm 10.1 \mathrm{~mm} \mathrm{Hg}$ ). Office BP was significantly correlated with home morning $\mathrm{BP}$ in each treatment group. However, correlation coefficients of office BP-home BP relationships were small in both groups $(r=0.19 \sim 0.27$ for baseline or posttreatment SBP, and $r=0.36 \sim 0.55$ for baseline or post-treatment $\mathrm{DBP})$, reflecting differences in timing and condition of $\mathrm{BP}$ measurements.

\section{DISCUSSION}

The primary endpoint in this study, home BP early in the morning after 8 weeks of treatment, was not significantly different between the telmisartan and valsartan groups. Change in morning SBP differed by only $0.9 \mathrm{~mm} \mathrm{Hg}$ between the telmisartan and valsartan groups (Table 3). However, s.d. of home BP was larger (that is, approximately $8 \mathrm{~mm} \mathrm{Hg}$ ) than the value we assumed for estimation of sample size, and type II error underlying the lack of inter-group difference in the primary endpoint cannot be excluded. Nevertheless, both valsartan and telmisartan significantly reduced morning SBP in patients on amlodipine, and BP control to the target home SBP $(<135 \mathrm{~mm} \mathrm{Hg})$ was achieved in $19-20 \%$ of the patients.

Interestingly, change in SBP with telmisartan treatment was correlated with baseline SBP level, whereas such a correlation was not found for valsartan (Figure 3). The reason for the different relationships between baseline BP level and change in BP after the 8 -week treatment in the telmisartan and valsartan groups is unclear. However, a plausible explanation is that the level of neurohumoral activation counteracting the drug-induced BP fall was higher in the valsartan group because of the shorter plasma half-life than in the telmisartan group.

Of the secondary endpoints, home BP levels before sleep were similar in the telmisartan and valsartan groups (Table 4). However, a significant difference was found in the other secondary endpoint, BP variability in the morning, between the two groups. Individual s.d. and CV of morning SBP were significantly increased by valsartan, but such adverse effects on SBP variability were not observed for telmisartan (Figure 4). Determinants of home BP variability (that is, age, gender, BMI, heart rate, variability of heart rate, length of hypertension treatment $)^{6,23}$ were comparable in the valsartan and telmisartan groups, and thus inter-group difference in BP variability changes after treatment is attributable to pharmacological difference 
in these two ARBs. A recent meta-analysis indicated that the effect of $\mathrm{ARBs}$ on 'inter-individual' variation of BP is neutral as is that of betablockers, although long-acting CCBs reduce BP variation. ${ }^{16}$ The present study showed that the effect on intra-individual variation of morning BP is not uniform in ARBs.

Accumulating evidence indicates that BP variability has a significant impact on clinical outcomes in patients with hypertension, ${ }^{4-9}$ although some indices of BP variation do not appear to predict cardiovascular events. ${ }^{24,25}$ Kikuya et al. ${ }^{5}$ reported that an increase in day-by-day variation of home BP by 1 between-subject s.d. was associated with a $20 \%$ increase in cardiovascular event risk in 2455 subjects in the Ohasama Cohort. Similarly, the risk of cardiovascular events was shown to increase by $4 \%$ per $1 \mathrm{~mm} \mathrm{Hg}$ in day-by-day variability of morning home $\mathrm{BP}$ in the Finn-Home Study. ${ }^{8}$ In contrast, Schutte et al. ${ }^{9}$ reported that visit-to-visit variability of home BP did not increase cardiovascular events in a general population. However, hypertensive patients in their study showed a significant association of large BP variability with an increase in composite cardiovascular events. ${ }^{9}$ Recently, Rothwell et $a .^{7}$ re-analyzed data in the UK-TIA and ASCOT-BPLA trials and showed that the risk of stroke was increased by 3-6-fold in patients with s.d. of SBP being in the top decile and that this impact of SBP variation on stroke risk was independent of mean SBP. Interestingly, the relationships between the risk of stroke and decile of s.d. of SBP were similar in amlodipine-treated and atenolol-treated patients, although patterns of distribution of patients across deciles of SBP s.d. were different between the two treatment groups (that is, the number of patients was larger in lower decile of SBP s.d. in the amlodipine-treated group and the pattern was the opposite in the atenolol-treated group). Collectively, these findings support the notion that variation of home BP should be minimized for the prevention of cardiovascular events in patients with hypertension.

In several earlier studies, efficacies of telmisartan and valsartan in BP control were directly compared. ${ }^{18-21,26}$ Reductions in 24-h mean, morning mean and daytime mean BPs were larger by 10 $15 \mathrm{~mm} \mathrm{Hg}$ in a group treated with $80 \mathrm{mg}$ of telmisartan than in a group treated with $80 \mathrm{mg}$ of valsartan. ${ }^{18}$ There was no significant difference in 24-h BP reduction between $80 \mathrm{mg}$ telmisartan and $160 \mathrm{mg}$ valsartan groups, although BP reduction during the last $6 \mathrm{~h}$ of the dosing period was larger in the telmisartan-treated group in a cross-over study by White et al.. ${ }^{21}$ In contrast, Calvo et al. ${ }^{19}$ reported that reduction in 24-h mean SBP was significantly larger by $19 \mathrm{~mm} \mathrm{Hg}$ in a group treated with $160 \mathrm{mg}$ of valsartan than in a group treated with $80 \mathrm{mg}$ of telmisartan. When combined with $25 \mathrm{mg}$ of hydrochlorothiazide, $80 \mathrm{mg}$ of telmisartan reduced SBP and DBP by 3 and $2 \mathrm{~mm} \mathrm{Hg}$ more, respectively, than $160 \mathrm{mg}$ of valsartan. ${ }^{26}$ In the present study, we examined, for the first time, efficacies of telmisartan and valsartan as an add-on therapy for patients on CCB. Although the two ARBs similarly reduced mean home $\mathrm{BP}$ in the morning, $\mathrm{BP}$ reduction by valsartan was accompanied by increased variability of SBP. Furthermore, telmisartan was more effective for $\mathrm{BP}$ reduction in a subgroup with higher baseline SBP. The presence and absence of superiority of one agent over another in the earlier studies and the present study,apparently,depends on the dose of each ARB and/or the clinical background of the study subjects, including pretreatment BP level. Nevertheless, the present study indicates that $40 \mathrm{mg}$ of telmisartan is preferable to $80 \mathrm{mg}$ of valsartan for control of morning home BP without an increase in BP variability in patients already being treated with amlodipine.

\section{CONFLICT OF INTEREST}

The authors declare no conflict of interest.

\section{ACKNOWLEDGEMENTS}

This study was supported by a grant-in-aid from the Waksman Foundation of Japan, (Tokyo, Japan).

1 Staessen JA, Asmar R, De Buyzere M, Imai Y, Parati G, Shimada K, Stergiou G, Redón J, Verdecchia PParticipants of the 2001 Consensus Conference on Ambulatory Blood Pressure Monitoring. Task Force II: blood pressure measurement and cardiovascular outcome. Blood Press Monit 2001; 6: 355-370

2 Giles TD. Circadian rhythm of blood pressure and the relation to cardiovascular events. I Hypertens Supp/ 2006; 24: S11-S16.

3 Pickering TG, Miller NH, Ogedegbe G, Krakoff LR, Artinian NT, Goff D, American Heart Association; American Society of Hypertension; Preventive Cardiovascular Nurses Association. Call to action on use and reimbursement for home blood pressure monitoring: executive summary: a joint scientific statement from the American Heart Association. Hypertension 2008; 52: 1-9.

4 Eto M, Toba K, Akishita M, Kozaki K, Watanabe T, Kim S, Hashimoto M, Ako J, lijima K, Sudoh N, Yoshizumi M, Ouchi Y. Impact of blood pressure variability on cardiovascular events in elderly patients with hypertension. Hypertens Res 2005; 28: 1-7.

5 Kikuya M, Ohkubo T, Metoki H, Asayama K, Hara A, Obara T, Inoue R, Hoshi H, Hashimoto J, Totsune K, Satoh $\mathrm{H}$, Imai Y. Day-by-day variability of blood pressure and heart rate at home as a novel predictor of prognosis: the Ohasama study. Hypertension 2008; 52: 1045-1050.

6 Kato T, Kikuya M, Ohkubo T, Satoh M, Hara A, Obara T, Metoki H, Asayama K, Hirose T, Inoue R, Kanno A, Totsune K, Hoshi H, Satoh H, Imai Y. Factors associated with dayby-day variability of self-measured blood pressure at home: the Ohasama study. Am J Hypertens 2010; 23: 980-986.

7 Rothwell PM, Howard SC, Dolan E, O'Brien E, Dobson JE, Dahlöf B, Sever PS, Poulter NR. Prognostic significance of visit-to-visit variability, maximum systolic blood pressure, and episodic hypertension. Lancet 2010; 375: 895-905.

8 Johansson JK, Niiranen TJ, Puukka PJ, Jula AM. Prognostic value of the variability in home-measured blood pressure and heart rate: the Finn-Home Study. Hypertension 2012; 59: 212-218.

9 Schutte R, Thijs L, Liu YP, Asayama K, Jin Y, Odili A, Gu YM, Kuznetsova T, Jacobs L, Staessen JA. Within-subject blood pressure level-not variability-predicts fatal and nonfatal outcomes in a general population. Hypertension 2012; 60: 1138-1147.

10 Kario K, Pickering TG, Umeda Y, Hoshide S, Hoshide Y, Morinari M, Murata M, Kuroda T, Schwartz JE, Shimada K. Morning surge in blood pressure as a predictor of silent and clinical cerebrovascular disease in elderly hypertensives: a prospective study. Circulation 2003; 107: 1401-1406.

11 Li Y, Thijs L, Hansen TW, Kikuya M, Boggia J, Richart T, Metoki H, Ohkubo T, Torp-Pedersen C, Kuznetsova T, Stolarz-Skrzypek K, Tikhonoff V, Malyutina S, Casiglia E, Nikitin Y, Sandoya E, Kawecka-Jaszcz K, Ibsen H, Imai Y, Wang J, Staessen JAlnternational Database on Ambulatory Blood Pressure Monitoring in Relation to Cardiovascular Outcomes Investigators. Prognostic value of the morning blood pressure surge in 5645 subjects from 8 populations. Hypertension 2010; 55 1040-1048.

12 Verdecchia P, Angeli F, Mazzotta G, Garofoli M, Ramundo E, Gentile G, Ambrosio G Reboldi G. Day-night dip and early-morning surge in blood pressure in hypertension prognostic implications. Hypertension 2012; 60: 34-42.

13 Redón J, Roca-Cusachs A, Mora-Maciá J. Uncontrolled early morning blood pressure in medicated patients: the ACAMPA study. Analysis of the Control of Blood Pressure using Abulatory Blood Pressure Monitoring. Blood Press Monit 2002; 7: 111-116.

14 Obara T, Ohkubo T, Asayama K, Kikuya M, Metoki H, Inoue R, Komai R, Murai K, Hashimoto J, Totsune K, Imai YJ-Home Study Group. Prevalence of masked hypertension in subjects treated with antihypertensive drugs as assessed by morning versus evening home blood pressure measurements: the J-HOME study. Clin Exp Hypertens 2008; 30: 277-287.

15 Nasothimiou EG, Tzamouranis D, Rarra V, Roussias LG, Stergiou GS. Diagnostic accuracy of home vs ambulatory blood pressure monitoring in untreated and treated hypertension. Hypertens Res 2012; 35: 750-755.

16 Webb AJ, Fischer U, Mehta Z, Rothwell PM. Effects of antihypertensive-drug class on interindividual variation in blood pressure and risk of stroke: a systematic review and meta-analysis. Lancet 2010; 375: 906-915.

17 Israili ZH. Clinical pharmacokinetics of angiotensin II (AT1) receptor blockers in hypertension. J Hum Hypertens 2000; 14(Suppl 1), S73-S86.

18 Littlejohn T, Mroczek W, Marbury T, VanderMaelen CP, Dubiel RF. A prospective, randomized, open-label trial comparing telmisartan $80 \mathrm{mg}$ with valsartan $80 \mathrm{mg}$ in patients with mild to moderate hypertension using ambulatory blood pressure monitoring. Can J Cardiol 2000; 16: 1123-1132.

19 Calvo C, Hermida RC, Ayala DE, Ruilope LM. Effects of telmisartan $80 \mathrm{mg}$ and valsartan $160 \mathrm{mg}$ on ambulatory blood pressure in patients with essential hypertension. J Hypertens 2004; 22: 837-846.

20 Galle J, Schwedhelm E, Pinnetti S, Böger RH, Wanner CVIVALDI investigators. Antiproteinuric effects of angiotensin receptor blockers: telmisartan versus valsartan 
in hypertensive patients with type 2 diabetes mellitus and overt nephropathy. Nephrol Dial Transplant 2008; 23: 3174-3183.

21 White WB, Lacourciere Y, Davidai G. Effects of the angiotensin II receptor blockers telmisartan versus valsartan on the circadian variation of blood pressure: impact on the early morning period. Am J Hypertens 2004; 17: 347-353.

22 Matsuo S, Imai E, Horio M, Yasuda Y, Tomita K, Nitta K, Yamagata K, Tomino Y, Yokoyama $\mathrm{H}$, Hishida ACollaborators developing the Japanese equation for estimated GFR. Revised equations for estimated GFR from serum creatinine in Japan. Am J Kidney Dis 2009; 53: 982-992.

23 Ishikura K, Obara T, Kato T, Kikuya M, Shibamiya T, Shinki T, Ikeda U, Kobayashi $Y$, Metoki H, Mano N, Kuriyama S, Ohkubo T, Imai YJ-HOME-Morning Study Group. Associations between day-by-day variability in blood pressure measured at home and

\section{APPENDIX}

The SPEED Study Committees and Investigators

Steering Committee:

Kazuaki Shimamoto, Tetsuji Miura, Shigeyuki Saitoh, Hideaki Yoshida (Sapporo Medical University), Hiroshi Oimatsu (Hakodate Goryokaku Hospital), Hitoshi Ooiwa (Oji General Hospital), Shigemichi Tanaka (Teine Keijinkai Hospital), Takatoshi Nishimiya (Japanese Asahikawa Red Cross Hospital), Manabu Hayashi (Hokkaido Obihiro Kohsei General Hospital), Takayuki Matsuki (Steel Memorial Muroran Hospital), Yoshihiro Mori (Saiseikai Otaru Hospital), Katsuhiro Higashiura (Nemuro City Hospital)

Event Assessment Committee:

Tetsu Yamaguchi (Toranomon Hospital), Satoshi Ogawa (International University of Health and Welfare), Kazuhisa Kodama (Amagasaki Central Hospital)

SPEED Investigators (non-committee members): antihypertensive drugs: the J-HOME-Morning study. Clin Exp Hypertens 2012; 34: 297-304.

24 Pierdomenico SD, Lapenna D, Bucci A, Manente BM, Mancini M, Cuccurullo F, Mezzetti A. Blood pressure variability and prognosis in uncomplicated mild hypertension. Am Heart J. 2005; 149: 934-938.

25 Pierdomenico SD, Lapenna D, Di Tommaso R, Di Carlo S, Esposito AL, Di Mascio R, Ballone E, Cuccurullo F, Mezzetti A. Blood pressure variability and cardiovascular risk in treated hypertensive patients. Am J Hypertens 2006; 19: 991-997.

26 White WB, Punzi HA, Murwin D, Koval SE, Davidai G, Neutel JM. Effects of the angiotensin II receptor blockers telmisartan vs valsartan in combination with hydrochlorothiazide $25 \mathrm{mg}$ once daily for the treatment of hypertension. J Clin Hypertens (Greenwich) 2006; 8: 626-633.

Munenori Furugen, Row Ishimoto, Norie Saito, Masatada Fukuoka, Koh Hamabe, Hideyuki Murakami, Motoya Nakagawa, Kuniyasu Kikuiri, Masahiro Nishihara, Satoshi Sato, Tetsuya Noto, Akihito Tsuchida, Katsuhisa Ishii, Michihiro Iwata, Taketoshi Komakine, Katsuo Suzuki, Yasuhiro Nakamura, Yukinaga Nozawa, Hironori Kobayashi, Nobuhiko Togashi, Tetsuro Shoji, Toshiaki Ando, Kazuaki Yamauchi, Toshiaki Kijima, Niro Sawai, Kimio Nishizato, Hitomi Yamamoto, Tomoaki Nakata, Takashi Koyanagi, Hiroyuki Hotta, Michifumi Kyuma, Tohru Ohtomo, Toshiyuki Tobisawa, Yasuyuki Shinshi, Shieri Miyazaki, Yoshito Ohnuma, Tohru Hasegawa, Akifumi Takada, Yoshiaki Terashima, Takuji Yoshioka, Tomoaki Matsumoto, Toshio Tsubokura, Yoshitoki Takagawa, Daisuke Yoshida, Kenichi Sakamoto, Yuichi Nakamura, Tomoya Koizumi, Makoto Hagiwara, Kazuyuki Urabe, Shigeru Marusaki, Shigeo Yoshida, Naofumi Kaneko, Nobuyuki Ura, Haruki Sasaki, Takashi Ise, Takahiro Nishitani, Mitsuhiro Nishimura, Mitsuhiro Togashi. 